\title{
Prevention of Experimental Myasthenia Gravis by Nasal Administration of Synthetic Acetylcholine Receptor T Epitope Sequences
}

\author{
Peter I. Karachunski, ${ }^{\star}$ Norma S. Ostlie, ${ }^{*}$ David K. Okita, ${ }^{\star}$ and Bianca M. Conti-Fine ${ }^{\star \ddagger}$ \\ $*$ Department of Biochemistry, College of Biological Sciences, University of Minnesota, St. Paul, Minnesota 55108, and ${ }^{\ddagger}$ Department of \\ Pharmacology, School of Medicine, University of Minnesota, Minneapolis, Minnesota 55455
}

\begin{abstract}
$T$ cell tolerization prevents and improves $T$ cell-mediated experimental autoimmune diseases. We investigated here whether similar approaches could be used for antibody (Ab)-mediated autoimmune diseases. Myasthenia gravis, caused by IgG Ab against muscle acetylcholine receptor (AChR), is perhaps the best characterized of them. We used an animal model, experimental myasthenia gravis induced in C57B1/6 mice by immunization with Torpedo acetylcholine receptor (TAChR), to demonstrate that nasal administration of synthetic sequences of the TAChR $\alpha$-subunitforming epitopes recognized by anti-TAChR CD4 ${ }^{+} \mathrm{T}$ helper cells (residues $\alpha 150-169, \alpha 181-200$, and $\alpha 360-378$ ), given before and during immunization with TAChR, causes decreased $\mathrm{CD}^{+}$responsiveness to those epitopes and to TAChR, reduced synthesis of anti-TAChR Ab, and prevented experimental myasthenia gravis. These effects were not induced by nasal administration of synthetic epitopes of diphtheria toxin.

Secretion of IL-2, IL-4, and IL-10 by spleen T cells from TAChR immunized mice, in response to challenge with TAChR in vitro, indicated that in sham-tolerized mice only Th1 cells responded to TAChR, while peptide-treated mice had also an AChR-specific Th2 response. The TAChR peptide treatment induced also in vitro anergy to the TAChR of the spleen T cells, which was reversed by IL-2. (J. Clin. Invest. 1997. 100:3027-3035.) Key words: acetylcholine receptor $\cdot$ autoimmunity $\cdot$ mice $\cdot$ nasal tolerance $\cdot \mathrm{T}$ cells
\end{abstract}

\section{Introduction}

Ideal treatments of autoimmune disease would specifically affect the autoreactive $\mathrm{T}$ and $\mathrm{B}$ cells. Specific tolerization of $\mathrm{T}$ cells can be obtained by delivery of the antigen $(\mathrm{Ag})^{1}$ through routes, such as oral, intraperitoneal and nasal administration, that downregulate, rather than activate, $\mathrm{CD}^{+}$responses(1-2).

Bianca M. Conti-Fine was previously known as Bianca M. ContiTronconi.

Address correspondence to Bianca M. Conti-Fine, Department of Biochemistry, University of Minnesota, 1479 Gortner Ave., St. Paul, MN 55108. Phone: 612-624-6796; FAX: 612-625-5780. E-mail: conti@ biosci.cbs.umn.edu

Received for publication 6 May 1997 and accepted in revised form 24 September 1997.

J. Clin. Invest.

(C) The American Society for Clinical Investigation, Inc. 0021-9738/97/12/3027/09 \$2.00

Volume 100, Number 12, December 1997, 3027-3035

http://www.jci.org
Tolerization of $\mathrm{T}$ cells by those procedures has proven effective for prevention and/or treatment of $\mathrm{CD} 4^{+} \mathrm{T}$ cell mediated autoimmune diseases like experimental autoimmune encephalomyelitis (EAE) (3-6). It is not clear if similar approaches could be used for Ab-mediated diseases for two reasons. First, those procedures, while effective at reducing Ag-specific $\mathrm{CD}^{+}{ }^{+}$responses, may directly stimulate the $\mathrm{B}$ cells specific for the administered $\mathrm{Ag}$ (7-10); this may have disastrous consequences, as it has been shown in marmoset EAE (5). Intraperitoneal administration of myelin resulted in $\mathrm{CD}^{+}$ tolerance to myelin, but also in a acute, fatal form of EAE, mediated by Ab specific for the myelin oligodendrocyte glycoprotein. Also, those procedures stimulate Th2 cells, that, while downregulating the proinflammatory Th1 cells, can stimulate Ab synthesis (10-11), and cause exacerbation rather than improvement of Ab-mediated autoimmune diseases. Short T epitope sequence may be safer for $\mathrm{T}$ cell tolerance procedures than the whole Ag molecule, since peptide-specific Ab very seldom cross react with the cognate native Ag (12). Short peptides have been successfully used for oral tolerization in EAE (13), but they are not ideal for that procedure because they are easily digested by the gastrointestinal proteases.

Nasal administration is a promising tolerizing route when using epitope peptides, since it does not need to overcome the proteolytic barriers present in the digestive system. Also, short peptides cross epithelia more easily than large proteins. Thus, synthetic $\mathrm{CD}^{+}{ }^{+}$epitope sequences may be more effective than the whole Ag for tolerance induction (3). Nasal administration of the auto-Ag before immunization induced tolerance to that $\mathrm{Ag}$, and prevented development of $\operatorname{EAE}(3,6)$, collagen-induced arthritis (6), experimental uveitis (14), and experimental myasthenia gravis (EMG) (15-17). Aerosol administration of myelin basic protein (MBP) to MBP-immunized rats that had developed relapsing EAE decreased the intensity of the immune response to MBP and the severity of the attacks (6). Spleen $\mathrm{T}$ cells from rats that had inhaled MBP transferred protection to naive animals (6).

Myasthenia gravis (MG) is caused by an autoimmune response to the muscle acetylcholine receptor (AChR), with sensitization of $\mathrm{CD}^{+}$cells and production of high affinity $\mathrm{IgG}$ (18). EMG is induced in rodents and rabbits by immunization with purified AChR from the electric organ of Torpedo fish (TAChR). This causes sensitization of $\mathrm{CD}^{+}$and $\mathrm{B}$ cells, and production of high affinity $\mathrm{IgG} \mathrm{Ab}$ which cross react with mouse muscle AChR and cause myasthenic symptoms (18). Although mice are much less prone to EMG than Lewis rats

1. Abbreviations used in this paper: $\mathrm{Ab}$, antibody; $\mathrm{AChR}$, acetylcholine receptor; Ag, antigen; $\alpha \mathrm{BTX}, \alpha$-bungarotoxin; DTX, diphteria toxin; EAE, experimental autoimmune encephalomyelitis; EMG, experimental myasthenia gravis; MG, myasthenia gravis; MBP, myelin basic protein; S.I., stimulation indexes; TAChR, Torpedo acetylcholine receptor. 
and rabbits, C57Bl/6 (B6) mice are susceptible to EMG (18). Their anti-TAChR CD4 ${ }^{+} \mathrm{T}$ cells recognize primarily epitopes within residues 146-169, 181-200, and 360-378 of the TAChR $\alpha$ subunit-the sequence $\alpha 146-169$ is the most immunogenic (18). Universal, immunodominant $\mathrm{CD}^{+}$epitopes exist on the AChR, recognized in all $\mathrm{MG}$ patients by $\mathrm{CD} 4^{+} \mathrm{T}$ cells able to drive the synthesis of pathogenic anti-AChR Ab (18). Thus, to demonstrate that nasal administration of immunodominant TAChR CD4 ${ }^{+}$epitopes affects the anti-TAChR response and the appearance of EMG, could pave the way for development of similar treatments for human MG. In these studies, we investigated the effect on EMG susceptibility and on the T cell and $\mathrm{Ab}$ responses to TAChR, of nasal administration to $\mathrm{B} 6$ mice of synthetic $\mathrm{CD} 4^{+}$epitope sequences of the TAChR $\alpha$

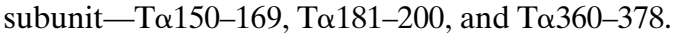

\section{Methods}

Peptide synthesis and characterization. We synthesized (19) and used three peptides, 19-20 residue long, corresponding to residues 150-169, 181-200, and 360-378 of the TAChR $\alpha$ subunit. The peptide codes include T $\alpha$ for TAChR $\alpha$ subunit and two numbers, indicating the position on the $\alpha$ subunit sequence of the first and last residues of the peptide. Also, we used three 20-residue peptides, synthesized with the same approach, and corresponding to the sequence regions 271-290, $321-340$, and 431-450 of diphtheria toxin (DTX), that previous studies have demonstrated to be highly and universally immunogenic for human $\mathrm{CD}^{+} \mathrm{T}$ cells (reference 20; Diethelm-Okita, Okita, and Conti-Fine, unpublished observations). We reported in detail previously the characterization of all those peptides (20-21).

Purification of TAChR. We purified TAChR from Torpedo californica electric organ as alkali-stripped TAChR-rich membrane fragments, characterized as we described previously (21). The TAChR concentration was determined as $\alpha$-bungarotoxin $(\alpha \mathrm{BTX})$ binding sites (22), the protein concentration by the Lowry et al. assay (23). The TAChR preparations we used contained 3.8-5.8 nmol of $\alpha$ BTX binding sites per $\mathrm{mg}$ protein. The protein composition was assessed by SDS-PAGE (24) that consistently showed, in the preparations we used, only the four TAChR subunits as the main protein bands. Occasional minor bands have low mol wt and are proteolytic products of the TAChR subunits (21). For use in cell cultures, we diluted the TAChR-rich membrane fragments in RPMI 1640 as needed, and sterilized them by UV irradiation. For immunization, we solubilized them in $1 \%$ Triton X-100 (21), diluted them to $0.5 \mathrm{mg} / \mathrm{ml}$ in PBS, and stored them at $-80^{\circ} \mathrm{C}$.

Mice and induction of tolerance. B6 mice were purchased from The Jackson Laboratory (Bar Harbor, ME) and housed at the animal facility of the University of Minnesota. After light anesthesia by i.m. injection of Ketaset $(100 \mathrm{mg} / \mathrm{kg}$; American Home Products Corp., Fort Dodge, IA), the mice received a total of $25 \mu \mathrm{l}$ of PBS containing $50 \mu \mathrm{g}$ of peptide $\mathrm{T} \alpha 150-169$ into both nostrils, either alone or pooled with equimolar amounts of peptides T $\alpha 180-200$ and T $\alpha 360-378$ (referred to as peptide pool or $\alpha$ pool). The dose we used was based on the results of a pilot experiment, in which we used increasing amounts of peptide $\alpha 150-169(50,100,200,400$, and $800 \mu \mathrm{g}$, respectively). The lowest dose $(50 \mu \mathrm{g})$ afforded a satisfactory level of protection, and we used that dose for further experiments. We administered the tolerogen as a solution instilled into the nostrils, instead of an aerosol, because it allowed accurate administration of defined amounts of solutions. Nasal delivery of either aerosol or liquid Ag solutions has similar efficacy in suppressing the effects of subsequent immunizations (6-14).

We used two nasal treatment schedules, to which we refer as protocols A and B. In protocol A, peptides or peptide-free PBS were administered $2 \mathrm{wk}$ before the first TAChR immunization, and then three more times, on the same day of the three immunizations with
TAChR (at 1 mo interval, see below). In protocol B, peptides or peptide-free PBS were administered weekly, starting two weeks before beginning of the immunization with TAChR, for a total of 14 treatments ( 2 before and 12 during TAChR immunization).

Control mice received $25 \mu \mathrm{l}$ of peptide-free PBS, or a pool of the three synthetic DTX peptides in PBS.

Immunizations. 8-10-wk-old mice were immunized by subcutaneous injections, along the back and at the base of the tail, of solubilized TAChR $(50 \mu \mathrm{g})$, or peptide T $\alpha 150-169(100 \mu \mathrm{g})$, or peptide pool (100 $\mu \mathrm{g}$ of each peptide). The mice were boosted twice at 4-wk intervals with the same amount of Ag. The Ag solutions (in $100 \mu \mathrm{l}$ PBS) were emulsified with an equal volume of complete Freund's adjuvant (FA) for the first injection, of incomplete FA for the boosts. Control mice were injected with PBS emulsified in the appropriate adjuvant.

Verification of the diffusion of nasally administered solutions into the mouse respiratory tract. We investigated which parts of the mouse respiratory system came in contact with solutions given nasally, using a solution of ethidium bromide. Ethidium bromide is a good marker because it is absorbed through the mucosal lining of the respiratory tract and it is brightly fluorescent under UV light. Two mice were anesthetized and $25 \mu \mathrm{l}$ of a $4 \%$ ethidium bromide solution in PBS was instilled into the nostrils, as described above. 10-15 min later the animals were killed by cervical dislocation. Their nasal cavities, larynx, trachea, bronchi, and lungs were dissected, washed in PBS and examined under UV light for ethidium bromide staining.

Evaluation of the clinical symptoms of EMG. EMG symptoms were quantified using a forced exercise by the inverted hang technique, sensitized by administration of a minute amount of pancuronium bromide $(0.03 \mathrm{mg} / \mathrm{kg}$ i.p.) just before the beginning of the test (25). The mice hung from a grid, and we measured the time it takes for the mouse to release its hold and fall three times (holding time). The test was performed on the day of the first nasal administration, on the day before each immunization, and 7-14 d after the third immunization, just before killing the animal. This test is parametric, and gives a quantitative assessment of the severity of the mouse weakness. To verify the myasthenic nature of the weakness we detected, mice with significant weakness were injected i.p. with the cholinesterase inhibitor edrophonium chloride (Reversol; Organon Inc., West Orange, NJ). Reversol immediately improved the strength of the animals, and alleviated the paralysis of the most severely affected mice. The test was performed blindly, i.e., without knowledge of the treatment that the mouse had received.

The holding time of normal mice was $10.4 \pm 2.1$ minutes $(n=99)$. Mice with holding times of 8 min or longer were considered normal, those with holding time $>4 \mathrm{~min}$ but $<8$ min were considered to have moderate symptoms, and those with holding time $<4$ min were considered severely affected. Mice that were paralyzed or had died of respiratory paralysis are represented in the figures as having holding time of zero.

Lymphocyte proliferation assay. 7-10 d after the last immunization, spleen $\mathrm{T}$ cells were purified from individual mice (21). Irradiated (3,000 rad) spleen cells from nonimmunized mice were diluted as needed in RPMI 1640 (GIBCO BRL, Gaithersburg, MD) supplemented with $10 \%$ heat inactivated FCS (GIBCO BRL), $50 \mu \mathrm{M} 2$-mercaptoethanol, $1 \mathrm{mM}$ L-glutamine, $10 \mathrm{mM}$ Hepes, $1 \mathrm{mM}$ sodium pyruvate, $100 \mathrm{U} / \mathrm{ml}$ penicillin, and $100 \mu \mathrm{g} / \mathrm{ml}$ streptomycin (culture medium $)$ and used as $\mathrm{Ag}$ presenting cells. The spleen T cells $\left(1 \times 10^{6} \%\right.$ $\mathrm{ml}$ in culture medium, $100 \mu \mathrm{l}$ per well) were seeded in triplicate in 96 flat-bottom well plates containing $100 \mu \mathrm{l}$ of $5 \times 10^{6} / \mathrm{ml} \mathrm{Ag}$ presenting cells. One of the following Ag was added: $10 \mu \mathrm{g} / \mathrm{ml}$ PHA (Sigma Chemical Co., St. Louis, MO); 5 or $10 \mu \mathrm{g} / \mathrm{ml} \mathrm{TAChR;} 5$ or $10 \mu \mathrm{g} / \mathrm{ml}$ of the individual epitope peptides. Controls were triplicate wells containing $\mathrm{T}$ and antigen presenting cells, without any Ag. After $4 \mathrm{~d}$, the cells were labeled for $16 \mathrm{~h}$ with $\left[{ }^{3} \mathrm{H}\right]$ thymidine $(1 \mu \mathrm{Ci}$ per well, specific activity $6.7 \mathrm{Ci} / \mathrm{mmol}$; Dupont-NEN, Boston, MA) and harvested (Titertek; Skatron, Inc., Sterling, VA). $\left[{ }^{3} \mathrm{H}\right]$ Thymidine incorporation was measured by liquid scintillation. The data are represented as 
stimulation indexes (S.I.), namely the ratio between the cpm obtained for a culture in the presence of a given stimulus, and the average cpm obtained for the unstimulated cultures (blanks). The blanks obtained for each experiments are indicated in the figure legends.

Determination of cytokine secretion in response to TAChR by mouse spleen cells in vitro. 7-10 d after the last immunization, spleen cells were cultured as described above for the proliferation assay, using sextuplicate cultures, with and without $10 \mu \mathrm{g} / \mathrm{ml}$ TAChR. Controls were triplicate cultures for each mouse group that did not receive any stimulus. After 12, 24, and $48 \mathrm{~h}$ the supernatants were harvested, and the IL-2, IL-4, and IL-10 concentrations were determined by capture ELISA using duplicate samples (PharMingen, San Diego, CA). We used anti-IL-2, anti-IL-4 and anti-IL-10 Ab, and recombinant IL-2, IL-4, and IL-10 (PharMingen) as standards, and followed the manufacturer's instructions.

Effect of preincubation with $I L-2$ on the response to TAChR by mouse spleen cells in vitro. Spleen cells from mice tolerized to the $\alpha$ pool after protocol B, or sham-tolerized with PBS, and immunized with TAChR as described above, were incubated in vitro with or without $1 \mathrm{ng} / \mathrm{ml}$ of mouse recombinant IL-2 (PharMingen) in culture medium for $5 \mathrm{~d}$ in 25-ml flasks (Costar, Cambridge, MA). The cells were then tested in the proliferation assay described above, using 5 and $10 \mu \mathrm{g} / \mathrm{ml}$ of TAChR.

Anti-AChR Ab assay. We obtained sera after each clinical testing. The serum concentration of anti-TAChR Ab was measured by radioimmuno precipitation assay (RIPA) using TAChR solubilized in Triton X-100 and labeled by the binding of $\left[{ }^{125} \mathrm{I}\right] \alpha \mathrm{BTX}$ (18). The $\mathrm{Ab}$ concentration is expressed as $\mu \mathrm{M}$ precipitated $\left[{ }^{125} \mathrm{I}\right] \alpha \mathrm{BTX}$.

Statistical analysis. The level of significance of the differences of the average responses between two groups was determined by two tailed Student's $t$ test, using Microsoft Excel (Microsoft Corp., Redmond, WA).

\section{Results}

Distribution in the respiratory tract of solutions administrated nasally. The mouse nostrils, larynx, and trachea were brightly stained by ethidium bromide administered with the same procedure as the peptide solutions. The staining was increasingly weaker in the bronchi, and only weak focal signals were present in the lung parenchyma (data not shown).

$T$ cells from mice treated nasally and immunized with TAChR peptides do not respond in vitro to the peptides, or to TAChR. To assess the effect of the nasal treatment with synthetic TAChR peptides on the ability of $\mathrm{CD}^{+}$cells to become sensitized to the same peptides, after they were administered as subcutaneous immunizing injections in adjuvant, we treated nasally three groups of mice with peptide T $\alpha 150-169$ or the $\alpha$ pool (50 $\mu \mathrm{g} /$ peptide), or peptide-free PBS, after protocol A. We immunized the mice three times with the peptide(s) used for the tolerization procedure, and 7-10 d after the last immunization we tested the spleen T cells of two mice tolerized with peptide T $\alpha 150-169$, four mice tolerized with the peptide pool and two sham-tolerized mice, for their proliferative response in vitro to the immunizing peptides and to the TAChR.

The results we obtained within each group were highly consistent. Fig. 1 reports the results obtained with one mouse from each group. The T cells of sham-tolerized mice had a good proliferative response in vitro to the immunizing peptide(s) and to TAChR, indicating that they recognize epitopes similar to those originating from TAChR processing (25), while the T cells of peptide-tolerized mice did not respond to the immunizing peptides(s) or to TAChR.

Nasal administration of synthetic AChR epitopes prevents
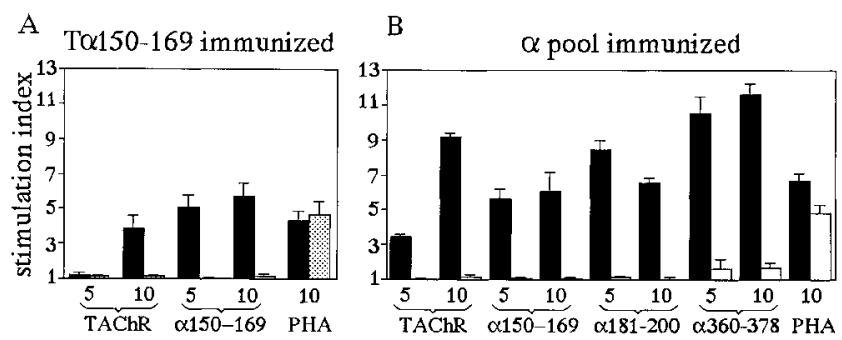

in vitro stimulant $(\mu \mathrm{g} / \mathrm{ml})$

Figure 1. Nasal administration of synthetic TAChR epitopes T $\alpha 150$ 169, T $\alpha 181-200$, and T $\alpha 360-378$ causes $\mathrm{T}$ cell unresponsiveness to those epitopes. Mice were given two nasal administrations of peptide T $150-169$ ( $A$, dotted columns), or $\alpha$ pool ( $B$, white columns), or peptide-free PBS (black columns) before immunization with the peptide(s) used for the nasal treatment. 7-10 d after the last immunization, we tested the proliferative response of spleen $\mathrm{T}$ cells to the immunizing peptide(s) and to TAChR. We report the results obtained for one mouse from each group, which are representative of those obtained for all mice of that group. We also report, for the sake of comparison, the response induced by $10 \mu \mathrm{g}$ of PHA. The columns represent the average S.I. of triplicate cultures. The average cpm obtained in the absence of any stimulation were $297 \pm 59$ in experiment A and 2,884 \pm 106 in experiment B. See text for experimental details.

appearance of EMG symptoms. Fig. 2 summarizes the results we obtained testing the strength of mice treated nasally with the peptide epitopes, and immunized with TAChR. We studied two groups of mice, treated with the TAChR peptide(s) as indicated or sham-tolerized $(P B S)$, using the protocol $\mathrm{A}(A)$ or protocol $\mathrm{B}(B)$. For each group we also report the results obtained for the same mice before TAChR immunization $(\mathrm{Na}$ ive). We report here the results obtained 8 or $10 \mathrm{wk}$ after beginning of the immunization (which were consistent), when we detected the maximum frequency of EMG symptoms.

In agreement with previous studies, that found variable EMG frequency (20-70\%) in TAChR immunized B6 mice (for review see reference 18), the frequency of EMG in the sham-tolerized groups varied. In a first pilot experiment (data not shown), 17 (89\%) of 19 mice developed EMG. In the two experiments reported in Fig. 2, all 5 sham-tolerized mice, and 5 of the 10 sham-tolerized mice had EMG symptoms, respectively.

When the tolerizing peptides were administered after protocol A, 5 of the 12 mice ( $42 \%$ ) tolerized with peptide T $\alpha 150$ 169 , and three of the eight mice $(37 \%)$ treated with the $\alpha$ pool after protocol A developed EMG, as compared with $100 \%$ of the mice sham-tolerized in parallel (Fig. $2 A$ ).

When the tolerizing peptides were administered after protocol $\mathrm{B}$, none of the mice that received nasal administration of peptide T $\alpha 150-169$ had detectable weaknesses, and one mouse in the group treated with the peptide pool had at $10 \mathrm{wk}$ a holding time barely below $8 \mathrm{~min} ; 50 \%$ of the sham-tolerized mice had EMG weakness (Fig. $2 \mathrm{~B}$ ).

Fig. 2 reports also the average holding time \pm SD of the groups of mice used in these experiments. In both experiments mice tolerized to peptide T $\alpha 150-169$ and to the $\alpha$ pool had significantly longer holding time than the sham-tolerized mice.

Reduced $T$ cell response to the sequence Ta150-169 and to 

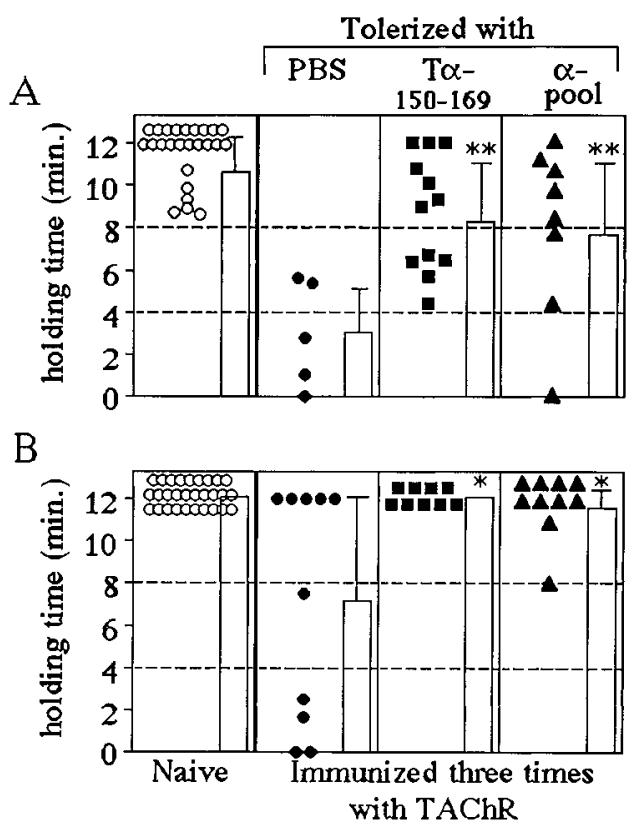

Figure 2. Nasal administration of synthetic TAChR CD4 $4^{+}$epitope peptides protects from EMG. Peptide T $\alpha 150-169$ or $\alpha$ pool or peptide-free PBS were administered nasally twice before immunization with TAChR, and at different time intervals during the course of the immunization ( $A$, monthly; $B$, weekly). Three immunizations with $50 \mu \mathrm{g}$ of TAChR, 1 mo apart, were also administered. We report the muscle strength of the mice, measured as holding time using the curare sensitized hanging test described in the text, after the third TAChR injection. We considered normal those mice having a holding time of 8 min or more, moderately sick those with holding time between 4 and $8 \mathrm{~min}$, and severely sick those with holding time of $<4$ $\min$. The 4 and 8 min levels are indicated by dashed horizontal lines. The panels labeled Naive report the values obtained for the mice used in these experiments before immunization with TAChR. The other plots reporting the results obtained for the mice sham-tolerized with PBS or tolerized with peptide T $\alpha 150-169$ or with a pool, as indicated above the plots. The average holding times \pm SD of the different groups are indicated, and the level of significance of the difference as compared with the sham-tolerized group $(* * P<0.002 ; * P<0.02)$. See text for experimental details.

the TAChR after immunization with TAChR of mice treated nasally with peptide T $\alpha 150-169$ or the $\alpha$ pool. We killed the mice used for the experiment reported in Fig. 2 B $10 \mathrm{wk}$ after beginning of the TAChR immunizations, and we tested the spleen $\mathrm{T}$ cells of each mouse in proliferation assays with TAChR and the individual peptides T $\alpha 150-169$, T $\alpha 181-200$, and T $\alpha 360-378$. The results are reported in Fig. 3, where the plots of the results are organized in four panels, according to the challenging Ag used in the proliferation assay. Each panel comprises the responses to that Ag obtained for mice shamtolerized (PBS), or tolerized to $\alpha 150-169$ or the $\alpha$ pool, as indicated below the individual plots. All but one of the sham-tolerized mice responded well to TAChR (S.I. = 10-29). Only eight mice are reported because two died of EMG before the experiment could be carried out. Thus, only three of the mice used in this experiment had detectable EMG when they were killed (black circles). Most peptide-treated mice responded to the TAChR. Their average responses (Fig. 3, horizontal bars) were slightly lower then those of the sham-tolerized group, but given the scattering of the data, the difference was significant only for the mice tolerized to the $\alpha$ pool. All groups of mice treated nasally with TAChR peptides had lower proliferative responses to the TAChR than the control mice sham-tolerized in parallel, but the extent of the reduction varied in the different groups. The particular groups of peptide-tolerized mice reported in Fig. 3 is representative of those that had the least reduction in proliferative response to TAChR. In most other groups, the reduction was much more substantial, and some group of $\alpha$ pool-tolerized mice had barely detectable or no proliferative responses to TAChR (e.g., see Figs. $5 B$ and 6).

The $T$ cells of most sham-tolerized mice responded to peptide T $\alpha 150-169$ but to a much lesser extent than to TAChR, because the anti-TAChR $\mathrm{CD}^{+}{ }^{+} \mathrm{T}$ cells of $\mathrm{B} 6$ mice recognize several epitopes on sequence regions other than T $\alpha 150-169$ (21). The T cells of both peptide-treated groups responded to To150-169 significantly less than the sham-treated mice; several mice did not respond to this epitope sequence (S.I. $<1.5)$.

Peptides T $\alpha 181-200$ and T $\alpha 360-378$, that are much less immunogenic for $\mathrm{CD}^{+}$cell sensitization than T $\alpha 150-169$ (23), were recognized poorly even by the spleen T cells of sham-tolerized mice. Previous reports demonstrated that the T cell response of B6 mice to those epitope sequences can be detected only when using purified $\mathrm{CD} 4^{+}$cells instead of total spleen $\mathrm{T}$ cells, as we did here (21). The response to peptides T $\alpha 181-$ 200 and T $\alpha 360-378$ of the $\alpha$ pool-tolerized mice was the same as that of the control mice. Thus, the reduced $\mathrm{T}$ cell recognition of the TAChR molecule of the mice tolerized with the peptide pool is at least partially due to a reduced response to epitopes formed by the sequence T $\alpha 150-169$.

The extent of the proliferative response to TAChR, T $\alpha 150$ 160 , and $\mathrm{T} \alpha 180-200$ of the sham-tolerized mice correlated

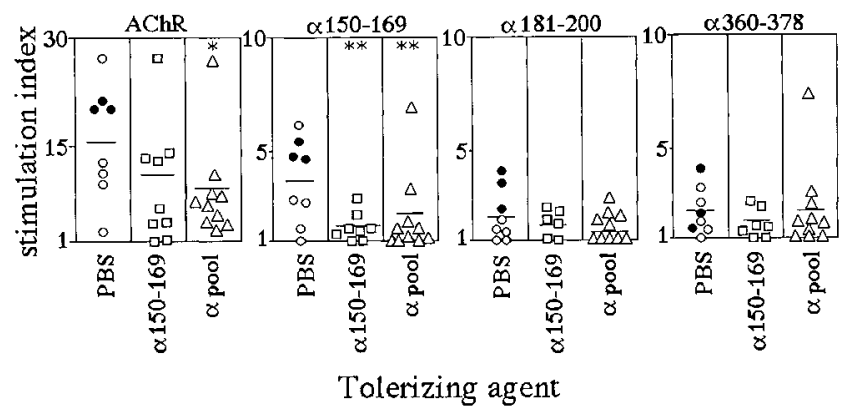

Figure 3. Spleen T cells from mice treated nasally with synthetic TAChR T epitope sequences and immunized with TAChR, respond minimally to peptide T $\alpha 150-169$ and respond to the TAChR to a lesser extent than the T cells from sham-tolerized controls. Mice received weekly nasal administrations of peptide-free PBS (circles), or T $\alpha 150-169$ (squares), or $\alpha$ pool (triangles) as indicated below each plot, and were immunized three times with TAChR. The spleen $T$ cells of individual mice were tested in proliferation assays with TAChR or the individual peptides T $\alpha 150-169$, T $\alpha 181-200$, and T $\alpha 360-378$, as indicated above each panel. The data are average S.I. \pm SD of triplicate cultures. The cpm in the absence of any stimulation were $190 \pm 88$. The proliferative responses of mice that had EMG are indicated with black circles. The average responses of the different groups, and the level of significance of the difference between peptide-tolerized and sham-tolerized mice are reported $(* P<0.03$; $* * P<0.01)$. See text for experimental details. 


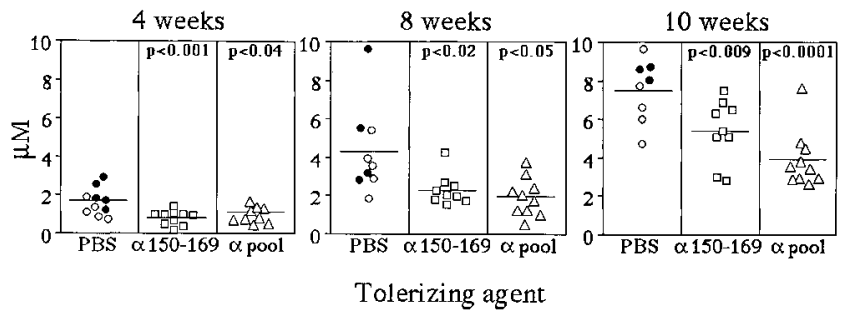

Figure 4. Mice treated nasally with TAChR peptides have less serum anti-TAChR Ab than sham-tolerized mice. Concentration of antiTAChR Ab in the sera of individual mice tolerized by weekly inhalations (protocol B) of peptide T $\alpha 150-169$ (squares) or peptide pool (triangles) or sham-tolerized with peptide-free PBS (circles), and immunized three times with TAChR, measured 4, 8, and 10 wk after the first TAChR immunization, as indicated above the plots. The $\mathrm{Ab}$ concentration is expressed as $\mu \mathrm{M}$ precipitated $\left[{ }^{125} \mathrm{I}\right] \alpha \mathrm{BTX}$ binding sites. Mice that presented EMG symptoms are indicated by black circles. The average $\mathrm{Ab}$ concentrations of the different groups and the level of significance of the difference between peptide-tolerized and sham-tolerized mice are indicated. See text for experimental details.

loosely with the presence of EMG symptoms: the three mice with EMG symptoms were among those with the highest S.I.

Nasal treatment with AChR peptides causes reduced synthesis of anti-TAChR Ab. We measured the serum anti-TAChR $\mathrm{Ab}$ concentration of individual mice tolerized with peptide T $\alpha 150-169$ or with the $\alpha$ pool or sham-tolerized 4,8 , and $10 \mathrm{wk}$ after the beginning of the immunization with TAChR (Fig. 4). Mice treated with T $\alpha 150-169$ or the $\alpha$ pool had significantly lower concentrations of anti-TAChR Ab than the shamtreated $(P B S)$ group as early as $4 \mathrm{wk}$ after the first TAChR immunization, although they eventually developed substantial concentrations of anti-TAChR Ab (at $10 \mathrm{wk} 5.5 \pm 1.5 \mu \mathrm{M}$ and $4.3 \pm 1.6 \mu \mathrm{M}$ versus $7.2 \pm 1.8 \mu \mathrm{M}$ in the sham-tolerized group). The anti-TAChR Ab concentration of individual sham-tolerized mice correlated loosely with the presence of EMG symptoms: mice with EMG symptoms were among those with highest Ab concentrations (Fig. 4, black circles).

Nasal administration of synthetic DTX peptides does not affect the anti-AChR T and antibody responses or development of EMG. To test the specificity of the effects observed after nasal administration of TAChR epitope peptides, we tested the effects on the anti-TAChR response and appearance of EMG of nasal administration of three DTX peptides, highly immunogenic for human $\mathrm{CD}^{+}$cells (20), of the same length and synthesized with the same procedure as the TAChR epitope sequences. The peptides were administered following protocol B, before and simultaneous with three immunizations with TAChR. At the same time, two other groups of mice were sham-tolerized with PBS or tolerized with the $\alpha$ pool. None of the $\alpha$ pool treated mice developed EMG, while the DTX peptide- and PBS-treated mice developed EMG with similar frequency ( $\sim 40 \%$ ) (Fig. $5 A$ and data not shown). Mice treated nasally with DTX peptide or PBS developed similar serum anti-AChR Ab concentrations, higher than those of the AChR peptide-tolerized mice (data not shown). After the third TAChR immunization, the spleen T cells of four mice from each group were pooled and tested for the proliferative response in vitro to TAChR. The spleen T cells from DTX peptide treated mice responded well to TAChR, while the re- sponses of spleen T cells of the $\alpha$ pool treated mice were consistently very low (Fig. 5 B).

The reduction of the in vitro response to TAChR of spleen $T$ cells from AChR peptides tolerized mice is reversed by $I L-2$. Anergy of Ag-specific $\mathrm{CD}^{+}{ }^{+} \mathrm{T}$ cells is a possible mechanism of $\mathrm{T}$ cell tolerization. A test of $\mathrm{T}$ cell anergy is reversal of the nonresponsiveness in vitro to the $\mathrm{Ag}$, by treatment of the $\mathrm{T}$ cells in vitro with IL-2 before Ag testing (26). Two groups of four mice each were treated nasally with PBS or with $\alpha$ pool after protocol B. After the third TAChR injection, the spleen $\mathrm{T}$ cells of the mice of each group were pooled and cultured with or without IL-2 as described in Methods. After the incubation, the IL-2 was removed and the spleen T cells thus treated were tested in proliferation assay for their responsiveness to the TAChR. For each condition and T cells population tested, we set up six independent cultures. Fig. 6 reports the average proliferation (measured as $\left[{ }^{3} \mathrm{H}\right]$ thymidine incorporation) of the sextuplicate cultures, set up with spleen T cells from $\alpha$ pool-tolerized or sham-tolerized mice, pretreated with or without IL-2, and cultivated in the absence of any stimulus, or in the presence of different concentrations of TAChR, as indicated. In the absence of IL-2 pretreatment, the spleen cells from $\alpha$ pool-tolerized mice responded to TAChR minimally, while those from sham-tolerized mice had a clear response. The IL-2 pretreatment increased to a small and nonsignificant extent the basal rate of cell proliferation of the T cells; it increased significantly $(P>0.001)$ the in vitro response to the TAChR of T cells from the sham-tolerized mice, and reversed the nonresponsiveness to the TAChR of the T cells from the $\alpha$ pool-tolerized mice. After IL-2 treatment, their spleen T cells responded to the TAChR to an extent comparable to that observed for IL-2 pretreated spleen T cells from the sham-tolerized mice (Fig. 6). The difference in $\left[{ }^{3} \mathrm{H}\right]$ thymidine incorporation induced by the presence of TAChR in the IL-2 pretreated $\mathrm{T}$ cells from the $\alpha$ pool-tolerized mice, as compared with that

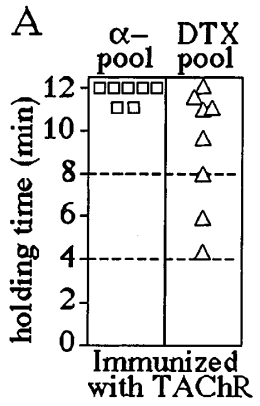

B

Figure 5. Nasal administration of synthetic DTX peptides does not affect the development of EMG or the anti-AChR T cell response. (A) muscle strength of individual mice, treated nasally with $\alpha$ pool or DTX peptides, as indicated above the plots, measured after the third TAChR injection as holding time using the curare sensitized hanging test. The 4 and $8 \mathrm{~min}$ levels are indicated by dashed horizontal lines. (B) Proliferative response to TAChR (5 and $10 \mu \mathrm{g}$, as indicated) of triplicate cultures of pooled spleen $\mathrm{T}$ cells of four mice from each group, after the third TAChR immunization (white columns, $\alpha$ pooltreated mice; black columns, DTX peptide-treated mice). The columns represent average S.I. \pm SD of triplicate cultures. The cpm in the absence of any stimulation were $228 \pm 29$ for the DTX peptide-tolerized mice, and $190 \pm 17$ for the $\alpha$ pool-tolerized mice. See text for experimental details. 

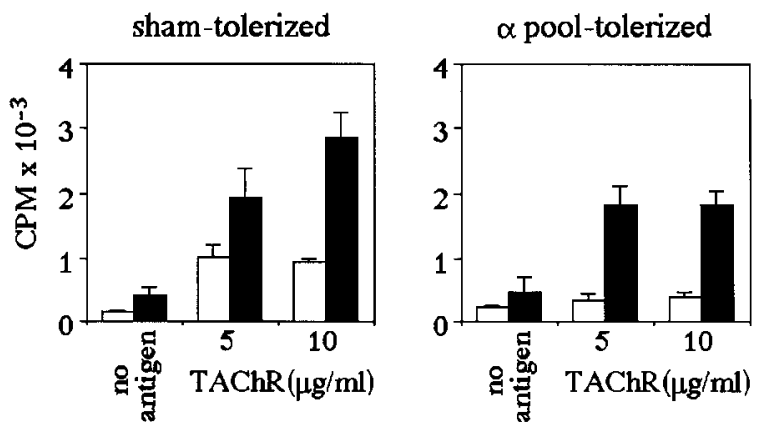

- pre-treated with IL-2

$\square$ pre-treated without IL-2

Figure 6. The reduction of the in vitro response to TAChR of spleen $\mathrm{T}$ cells from AChR peptides-tolerized mice is reversed by IL-2 pretreatment. After the third TAChR injection, spleen T cells of four mice sham-tolerized or tolerized with the $\alpha$ pool, as indicated above the panels, were pooled and incubated with (black columns) or without (white columns) IL-2. After the incubation, the IL-2 was removed and the cells were tested in proliferation assay for their response to TAChR. The columns represent the average proliferation (measured as $\left[{ }^{3} \mathrm{H}\right]$ thymidine incorporation) of six independent cultures, cultivated in the absence of any stimulation (no antigen) or in the presence of 5 and $10 \mu \mathrm{g} / \mathrm{ml}$ of TAChR, as indicated. See text for experimental details.

of the same cell population non-pretreated with IL-2 was highly significant $(P<0.00003)$.

Nasal treatment with AChR peptides stimulates AChR-specific Th2 cells. Stimulation of modulatory Th2 cells is another possible mechanism of peripheral tolerance. To test this possibility we investigated the secretion of IL-2, IL-4, and IL-10 in response to challenge with $\mathrm{TAChR}$, as representative cytokine for the Th1 (IL-2) and the Th2 (IL-4 and IL-10) subset. We tested the same mice treated nasally with PBS or with $\alpha$ pool after protocol B, that were used for the experiments on the effect of IL-2 treatment in vitro on the anti-TAChR responsiveness of spleen T cells, described above. After the third TAChR injection the spleen $\mathrm{T}$ cells of four mice of each group were pooled. For each of the pooled populations (sham-tolerized and $\alpha$ pool-tolerized) we set up sets of three (for IL-2 and IL-10) or two (for IL-4) independent cultures, that were cultivated with TAChR $(10 \mu \mathrm{g} / \mathrm{ml})$. Identical sets of parallel cultures cultivated without any stimuli served as controls for basal secretions of the interleukins studied. The concentration of IL-2, IL-4, and IL-10 was measured in the supernatants of the TAChR-stimulated and the control nonstimulated cultures, at different time interval after addition of the TAChR. Two independent assays were carried out for each supernatant and for each interleukin. The amount of IL-2 in the media was maximal $24 \mathrm{~h}$ after AChR addition, that of IL-4 and IL-10 at $48 \mathrm{~h}$ (data not shown). The averages of the data obtained in the two independent assays of the supernatants of three (for IL-2 and IL-10) or two (for IL-4) independent cultures of pooled spleen T cells exposed to TAChR for $24 \mathrm{~h}$ for IL-2 and for $48 \mathrm{~h}$ for IL-4 and IL-10, as compared with the concentration of the same interleukins in the supernatants of the parallel control cultures cultivated without antigenic stimuli, are reported in Fig. 7. The presence of TAChR induced the same modest but significant increase of IL-2 secretion in the $\alpha$ pool- and sham-tolerized groups. The presence of TAChR caused a modest increase in the IL-4 secretion, and no increase in the IL-10 secretion by the $\mathrm{T}$ cells from the sham-tolerized mice, while it caused a large increase in the secretion of both those interleukins in the $\alpha$ pool-tolerized group, that was significantly higher than the secretion of the same interleukins by the TAChR-stimulated T cells of the sham-tolerized mice (Fig. 7).

\section{Discussion}

We demonstrate here that nasal administration of a 20 residue TAChR synthetic peptide, T $\alpha 150-169$, that forms an immunodominant epitope recognized by pathogenic $\mathrm{CD}^{+}$cells, effectively protected B6 mice from induction of EMG caused by immunization with TAChR. The treatment was effective when administered before and during immunization with TAChR: monthly or weekly administrations had comparable effects. This suggests that nasal administration did not cause further priming of pathogenic anti-AChR $\mathrm{CD}^{+} \mathrm{T}$ cells. Protection from EMG was associated with reduced $\mathrm{T}$ cell reactivity in vitro to the TAChR, reduced levels of anti-TAChR Ab in the blood, and minimal or absent proliferative response of spleen T cells to the immunodominant peptide T $\alpha 150-169$. These effects were Ag-specific, since they could not be reproduced by nasal administration of comparable peptide sequences of DTX.

Although nasal administration of peptides T $\alpha 181-200$ and T $\alpha 360-378$ affected subsequent sensitization of $\mathrm{T}$ cells to all those sequences (Fig. 1), the protective effects on EMG induction observed here are likely due to tolerization of $\mathrm{CD} 4^{+}$cells that recognize epitopes within the sequence T $\alpha 150-169$, because nasal administration of peptide T $\alpha 150-169$ alone was as
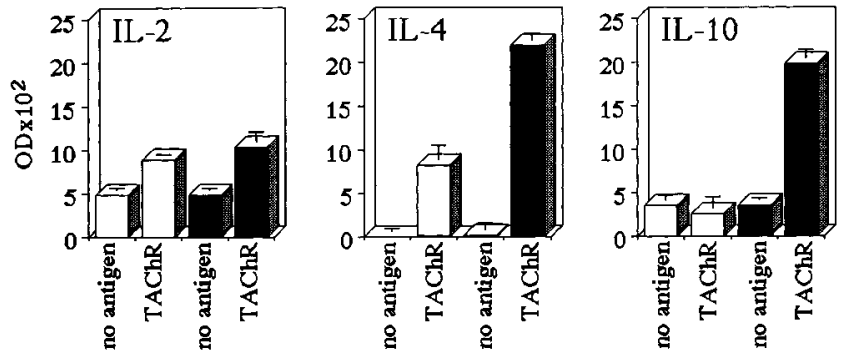

$\square$ sham-tolerized $\square \propto$ pool-tolerized

Figure 7. Nasal treatment with AChR peptides stimulates AChR specific Th2 cells. Secretion of IL-2, IL-4, and IL-10 in response to challenge with TAChR by spleen T cells of four mice treated nasally (protocol B) with PBS (white columns) or $\alpha$ pool (black columns), after three TAChR injections. Pooled spleen T cells from four mice for each group were used to set up triplicate (for the test of IL-2 and IL-10 secretion) or duplicate (for the test of IL-4 secretion) independent cultures, that were cultivated with $10 \mu \mathrm{g} / \mathrm{ml}$ of TAChR. Identical sets of parallel cultures that did not receive any stimulus served as controls for basal secretions of the interleukins studied (no antigen). The columns represent the averages of duplicate independent assays for each culture and for each condition, of supernatant samples obtained $24 \mathrm{~h}$ after TAChR addition to the culture for IL-2, $48 \mathrm{~h}$ for IL-10 ( $n=6$ for IL-2 and IL-10, $n=4$ for IL-4). The data are expressed as optical density units detected in ELISA. See text for experimental details. 
effective as administration of the $\alpha$ pool in protecting from EMG and reducing the $\mathrm{T}$ and $\mathrm{B}$ cell responses to TAChR.

Since the AChR destruction and dysfunction that results in EMG symptoms is caused by $\mathrm{Ab}$ binding, it is likely that the altered anti-TAChR $\mathrm{CD}^{+}{ }^{+}$reactivity after nasal tolerization results in protection from EMG because of a change in the anti-AChR Ab repertoire, because of preferential cooperation of different pairs of $\mathrm{CD} 4^{+}$helper T cells and B cells (27-29). In indirect support of this possibility, we found that mice tolerized with TAChR peptides, while protected from EMG, developed substantial amounts of anti-AChR Ab, but significantly lower than those observed for the mice sham-tolerized with peptide-free PBS (Fig. 4), or treated with DTX peptides. The pathogenic $\mathrm{Ab}$ missing in the TAChR peptide-tolerized mice are likely synthesized with the help of $\mathrm{CD}^{+}$cells recognizing epitopes within the sequence T $\alpha 150-169$. An important pathogenic role in mouse EMG of $\mathrm{CD}^{+}$cells recognizing epitopes within the sequence is supported by several findings: (a) neonatal tolerization to this sequence region reduces susceptibility to EMG (30 and Karachunski, P., and B.M. Conti-Fine, unpublished observation); (b) B6 mice primed with AChR and boosted with a synthetic sequence $\alpha 146-162$ developed EMG while mice boosted with a control peptide did not (31); and (c) in congenic B6 strains carrying the bm12 mutation of the I-A molecule, the ability by $\mathrm{CD} 4^{+}$cells to recognize this sequence correlates with propensity to EMG $(25,32-33)$. That $\mathrm{CD} 4^{+}$ cells sensitized to a single dominant $\mathrm{AChR}$ epitope may drive the synthesis of pathogenic anti-AChR Ab has been shown by transfer experiments for both rat (34) and human (35) $\mathrm{CD} 4^{+}$ lines against defined AChR epitopes.

Several mechanisms are involved in oral tolerance, including: anergy or deletion by apoptosis of $\mathrm{Ag}$ specific $\mathrm{T}$ cells, and induction of $\mathrm{Ag}$ specific regulatory $\mathrm{CD} 4^{+} \mathrm{Th} 2$ cells (36-37). In EAE, the same $\mathrm{CD} 4^{+}$precursors can develop into regulatory Th2 cells if the Ag is administered orally, or encephalitogenic Th1 cells if the Ag is administered subcutaneously in adjuvants (38). Ag-specific regulatory $\mathrm{CD}^{+}$cells may exert a nonspecific downregulating activity through secretion of cytokine, such as IL-4, IL-10, and TGF- $\beta$, that act on Th1 cells in topographic proximity, irrespective of their $\mathrm{Ag}$ specificity $(\mathrm{Ag}$ driven bystander suppression) (36). Oral administration of an $\mathrm{Ag}$ can induce tolerance by different mechanisms, depending upon the dose of $\mathrm{Ag}$ that was fed (39-40). Low doses of $\mathrm{Ag}$ generate $\mathrm{Th} 2$ regulatory cells, whereas high doses induce anergy (39-40) and/or apoptosis of Ag-reactive Th1 and Th2 cells (37). Given the functional similarity of the lymphoid tissues associated with the respiratory and the gastrointestinal systems, similar mechanisms are likely involved in nasal tolerance $(7,10)$. Studies on the protecting effect of nasal treatment with TAChR in the rat model of EMG have provided direct evidence for suppression of both Th1 and Th2 responses after nasal administration of the TAChR molecule, that was likely due to TGF- $\beta$-secreting cells (15-17).

Both clonal anergy and sensitization of regulatory Th2 cells seem to have occurred in our system (Figs. 6 and 7). High dose clonal deletion by apoptosis is less likely, since the highest doses we used were as effective as the lowest (50 $\mu$ g, i.e., 20 nmol). This dose compares in weight to those used for low dose oral tolerance (39). However, most $\mathrm{Ag}$ used in those studies had higher molecular weight than our peptides, and therefore their molar concentration would have been lower than that used for our peptide tolerization procedures. Epitope- specific anergy induction by nasal treatment with the TAChR peptides is directly supported by the finding that the reduced responsiveness in vitro of $\mathrm{T}$ cells to TAChR could be reversed by treatment with IL-2 (Fig. 6).

Anergy or deletion of the $\mathrm{T}$ cells recognizing epitopes within the sequence T $\alpha 150-169$ might suffice to protect from EMG, because, as discussed above, in $\mathrm{B} 6$ mice the $\mathrm{CD} 4^{+}$cells recognize epitopes within this sequence region are uniquely pathogenic. Also, the $\mathrm{CD}_{4}^{+}$response of $\mathrm{B} 6$ mice hyperimmunized with TAChR, which have EMG with high frequency, focuses almost exclusively on the sequence Ta150-169, rather than spreading to other TAChR epitopes (41). Thus, sensitization of $\mathrm{CD}^{+}$cells to epitopes within this sequence suffices to, and is prominent for, driving a pathogenic anti-TAChR Ab response. This is different from EAE, where progression of the disease correlates with spreading of the $\mathrm{CD}^{+}$response to new epitopes within MBP and other myelin components (42-43).

Nasal administration of TAChR peptides sensitized AChRspecific $\mathrm{Th} 2$ cells, which were not detectable after TAChR immunization in mice sham-tolerized or tolerized to DTX peptides (Fig. 7). On the other hand, TAChR immunization per se appeared to sensitize Th1 cells only (Fig. 7). Also in human MG Th1 cells are likely involved in the pathogenic anti-AChR response (44). In EAE, Th1 cells are the direct effectors of demyelinization, and their anergy or down regulation by the Th2 subset affects their pathogenic action directly, and has therapeutic effects (45). On the other hand, in EMG the protective effects of nasal administration of TAChR are indirect, and the procedures described here will not have a therapeutic effect when the tolerogenic peptides are administered only after establishment of the pathogenic anti-TAChR Ab response, given the long $\mathrm{Ab}$ life and the long life span of activated $\mathrm{B}$ cells (46) relative to the time frame of the experiments we describe here.

The use of $\mathrm{T}$ cell epitope peptides instead of the whole $\mathrm{Ag}$ avoids the risk that the nasally administered $\mathrm{Ag}$ will prime synthesis of pathogenic $\mathrm{Ab}$ - even if nasal administration of peptides causes production of anti-peptide $\mathrm{Ab}$, they are extremely unlikely to cross react with the cognate native $\mathrm{Ag}$ (12). That immunization with short TAChR peptides does not result in appearance of EMG has been shown in several studies (for review see reference 18 ). Also, short synthetic peptides are easily made, while scarce human auto-Ag cannot be purified from tissues and, if they are complex proteins, their production as recombinant protein might be difficult and labor intensive.

Nasal tolerization using the approach described here requires knowledge of the autoAg sequences forming $\mathrm{CD} 4^{+}$ epitopes; this might be difficult to do for human patients. This problem may not apply to $\mathrm{MG}$ because the $\mathrm{CD} 4^{+}$cells of most MG patients recognize a limited number of epitope sequences of the human AChR (for review see reference 18). Those sequence regions are recognized with high precursor frequency (44), and should therefore be considered both immunodominant and universal $\mathrm{CD} 4^{+}$epitopes. They would be ideal candidates for application to human MG of the approach described here. The presence on a protein $\mathrm{Ag}$ of a few immunodominant, universal epitope sequences for sensitization of human $\mathrm{CD} 4^{+}$cells occurs also for the normal responses to exogenous $\mathrm{Ag}$, like tetanus and diphtheria toxoid (20,45-49).

Although the procedure described here affects the antiAChR Ab-secreting B cells indirectly, and it does not have immediate therapeutic effects on established EMG, it could be a 
viable candidate for MG management, if associated to plasmapheresis and azathioprine, which eliminate the existing anti$\mathrm{AChR} \mathrm{Ab}$ and affect the activated $\mathrm{B}$ cells. The combined effects of such a two-pronged approach might result in a longlasting downregulation of the anti-AChR response, in both the $\mathrm{CD}^{+}$and the B cell compartments.

\section{Acknowledgments}

This work was partially supported by the National Institute of Neurological and Communicative Disorders and Stroke grant NS 23919 and by a research grant from the Council of Tobacco Research U.S.A., Inc. (B.M. Conti-Fine).

\section{References}

1. Matzinger, P. 1994. Tolerance, danger, and the extended family. Аnnu. Rev. Immunol. 12:991-1045.

2. Nossal, G. 1995. Choices following antigen entry: antibody formation or immunologic tolerance? Annu. Rev. Immunol. 13:1-27.

3. Metzler, B., and D.C. Wraith. 1993. Inhibition of experimental autoimmune encephalomyelitis by inhalation but not oral administration of the encephalitogenic peptide: influence of MHC binding affinity. Int. Immunol. 5: 1159-1169.

4. Miller, A., O. Lider, O. Abramsky, and H.L. Weiner. 1994. Orally administered myelin basic protein in neonates primes for immune responses and enhances experimental autoimmune encephalomyelitis in adult animals. Eur. $J$. Immunol. 24:1026-1032.

5. Genain, C.P., K. Abel, N. Belmar, F. Villinger, D.P. Rosenberg, C. Linington, C.S. Raine, and S.L. Hauser. 1996. Late complications of immune deviation therapy in a nonhuman primate. Science. 274:2054-2057.

6. Al-Sabbagh, A., P. Nelson, Y. Akselband, R. Sobel, and H. Weiner. 1996. Antigen-driven peripheral immune tolerance-suppression of experimental autoimmune encephalomyelitis and collagen-induced arthritis by aerosol administration of myelin basic protein or type II collagen. Cell. Immunol. 171:111-119.

7. Kuper, C., P. Koornstra, D. Hameleers, J. Biewenga, B.J. Spit, A.M. Duijvestijn, P.J. van Breda Vriesman, and T. Sminia. 1992. The role of nasopharyngeal lymphoid tissue. Immunol. Today. 13:219-224.

8. Liu, L., and G.G. MacPherson. 1993 Antigen acquisition by dendritic cells: intestinal dendritic cells acquire antigen administered orally and can prime naive T cells in vivo. J. Exp. Med. 177:1299-1307.

9. Husby, S., J. Mestecky, Z. Moldoveanu, S. Holland, and C. Elson. 1994. Oral tolerance in humans. $\mathrm{T}$ cell but not B cell tolerance after antigen feeding. J. Immunol. 152:4663-4670.

10. Neutra, M., E. Pringult, and J. Kraehenbuhl. 1996. Antigen sampling across epithelial barriers and induction of mucosal immune response. Ann. Rev. Immunol. 14:275-300.

11. Abbas, A.K., K.M. Murphy, and A. Sher. 1996. Functional diversity of helper T lymphocytes. Nature. 383:787-793.

12. Conti-Fine, B.M, K.E. McLane, and S. Lei. 1996. Antibodies as a tool to study the structure of membrane proteins. The case of the nicotinic receptor. Annu. Rev. Biophys. Biomol. Struct. 25:197-229.

13. Karpus, W.J., K.J. Kennedy, W.S. Smith, and S.D. Miller. 1996. Inhibition of relapsing experimental autoimmune encephalomyelitis in SJL mice by feeding the immunodominant PLP 139-151 peptide. Neurosci. Res. 45:410-423.

14. Dick, A., Y. Cheng, A. McKinnon, J. Liversidge, and J. Forrester. 1993. Nasal administration of retinal antigens suppresses the inflammatory response in experimental allergic uveoretinitis. A preliminary report of intranasal induction of tolerance with retinal antigens. Brit. J. Ophthalmol. 77:171-175.

15. Ma, C., G. Zhang, B. Xiao, J. Link, T. Olsson, and H. Link. 1995. Suppression of experimental autoimmune myasthenia gravis by nasal administration of acetylcholine receptor. J. Neuroimmunol. 58:51-60.

16. Ma, C., G. Zhang, B. Xiao, and H. Link. 1996. Cellular mRNA of interferon-gamma (IFN-gamma), IL-4 and transforming growth factor-beta (TGFbeta) in rats nasally tolerized against experimental autoimmune myasthenia gravis (EAMG). Clin. Exp. Immunol. 104:509-516.

17. Ma, C., G. Zhang, B. Xiao, Z.Y. Wang, J. Link, T. Olsson, and H. Link. 1996. Mucosal tolerance to experimental autoimmune myasthenia gravis is associated with down-regulation of AChR-specific IFN-gamma-expressing Th1like cells and up-regulation of TGF-beta mRNA in mononuclear cells. Ann. N.Y. Acad. Sci. 778:273-287.

18. Conti-Fine, B.M., M.P. Protti, M. Bellone, and J.F. Howard, Jr. 1997. Myasthenia Gravis: The Immunobiology of an Autoimmune Disease. R.G. Landes Company, Austin, Texas. 230 pp.

19. Houghten, R. 1985. General method for the rapid solid phase synthesis of large numbers of peptides: specificity of antigen-antibody interaction at the level of individual amino acids. Proc. Natl. Acad. Sci. USA. 82:5131-5135.
20. Raju. R., D. Navaneetham, D. Okita, B. Diethelm-Okita, D. McCormick, and B. Conti-Fine. 1995. Epitopes for human CD41 cells on diphtheria toxin: structural features of sequence segments forming epitopes recognized by most subjects. Eur. J. Immunol. 25:3207-3214.

21. Bellone, M., N. Ostlie, S. Lei, and B. Conti-Tronconi. 1991. Experimental myasthenia gravis in congenic mice. Sequence mapping and $\mathrm{H}-2$ restriction of $\mathrm{T}$ helper epitopes on the a subunits of Torpedo californica and murine acetylcholine receptors. Eur. J. Immunol. 21:2303-2310.

22. Schmidt, J., and M. Raftery. 1973. A simple assay for the study of solubilized acetylcholine receptors. Anal. Biochem. 52:349-354.

23. Lowry, O., N. Rosebrough, A. Farr, and R. Randall. 1981. Protein measurement with Folin phenol reagent. J. Biol. Chem. 193:256.

24. Laemmli, U. 1970. Cleavage of structural proteins during the assembly of the head of bacteriophage T4. Nature. 227:680-685.

25. Karachunski, P., N. Ostlie, M. Bellone, A. Infante, and B. Conti-Fine. 1995. Mechanisms by which the I-Abm12 mutation influences susceptibility to experimental myasthenia gravis: a study in homozygous and heterozygous mice. Scand. J. Immunol. 42:215-225.

26. DeSilva, D.R., K.B. Urdahl, and M.K. Jenkins. 1991. Clonal anergy is induced in vitro by $\mathrm{T}$ cell receptor occupancy in the absence of proliferation. $J$. Immunol. 147:3261-3267.

27. Palmer, M., and E. Sercarz. 1989. Determinant preferences in the relationship between T and B cell specific for lysozyme. In The Immune Response to Structurally Defined Proteins: The Lysozyme Model. S. Smith-Gill, and E. Sercarz, editors. Academic Press, New York. 285-321.

28. Myers, C.D. 1991. Role of B cell antigen processing and presentation in the humoral immune response. FASEB (Fed. Am. Soc. Exp. Biol.) J. 5:25472553.

29. Bellone, M., P. Karachunski, N. Ostlie, S. Lei, and B. Conti-Tronconi. 1994. Preferential pairing of $T$ and $B$ cells for production of antibodies without covalent association of T and B epitopes. Eur. J. Immunol. 24:799-804.

30. Shenoy, M., M. Oshima, M. Atassi, and P. Christadoss. 1993. Suppression of experimental autoimmune myasthenia gravis by epitope-specific neonatal tolerance to synthetic region alpha 146-162 of acetylcholine receptor. Clin Immunol. Immunopathol. 66:230-238.

31. Shenoy, M., E. Goluszko, and P. Christadoss. 1994. The pathogenic role of acetylcholine receptor a chain epitope with in a146-162 in the development of experimental autoimmune myasthenia gravis in C57Bl/6 mice. Clin. Immunol. Immunopathol. 73:1-6.

32. Bellone, M., N. Ostlie, S. Lei, X.-D. Wu, and B. Conti-Tronconi. 1991 The I-A bm12 mutation, which confers resistance to experimental myasthenia gravis, drastically affects the epitope repertoire of murine CD41 cells sensitized to nicotinic acetylcholine receptor. J. Immunol. 147:1484-1491.

33. Infante, A., P. Thompson, K. Krolik, and K. Wall. 1991. Determinant selection in murine experimental autoimmune myasthenia gravis: effect of the bm12 mutation on T-cell recognition of acetylcholine receptor epitopes. J. Immunol. 146:2977-2982.

34. Yeh, T.M., and K.A. Krolick. 1990. T cells reactive with a small synthetic peptide of the acetylcholine receptor can provide help for a clonotypically heterogeneous antibody response and subsequently impaired muscle function. J. Immunol. 144:1654-1660.

35. Conti-Fine, B.M., B. Diethelm-Okita, P. Karachunski, J. Howard, Jr., D. Okita, D. Navanitham, R. Raju, and Z.-Y. Wang. 1997. T-cell recognition of the Acetylcholine receptor in myasthenia gravis. Ann. N.Y. Acad. Sci. In press.

36. Weiner, H., A. Friedman, A. Miller, S.J. Khoury, A. Al-Sabbagh, L. Santos, M. Sayegh, R.B. Nussenblatt, D.E. Trenthan, and A.D. Hafler. 1994. Oral tolerance: immunologic mechanisms and treatment of animal and human organ-specific autoimmune diseases by oral administration of autoantigens. Annu. Rev. Immunol. 12:809-837.

37. Chen, Y., J. Inobe, R. Marks, P. Gonnella, V. Kuchroo, and H. Weiner. 1995. Peripheral deletion of antigen-reactive T cells in oral tolerance. Nature. 376:177-180.

38. Chen, Y., J. Inobe, V. Kuchroo, J. Baron, C.J. Janeway, and H. Weiner. 1996. Oral tolerance in myelin basic protein T-cell receptor transgenic mice: suppression of autoimmune encephalomyelitis and dose-dependent induction of regulatory cells. Proc. Natl. Acad. Sci. USA. 93:388-391.

39. Friedman, A., and H.L. Weiner. 1994. Induction of anergy or active suppression following oral tolerance is determined by antigen dosage. Proc. Natl. Acad. Sci. USA. 91:6688-6692.

40. Gregerson, D., W. Obritsch, and L. Donoso. 1993. Oral tolerance in experimental autoimmune uveoretinitis. Distinct mechanisms of resistance are induced by low dose vs. high dose feeding protocols. J. Immunol. 151:5751-5761.

41. Bellone, M., N. Ostlie, P. Karachunski, A. Manfredi, and B. Conti-Tronconi. 1993. Cryptic epitopes on the nicotinic acetylcholine receptor are recognized by autoreactive CD41 cells. J. Immunol. 151:1025-1038.

42. McRae, B., C. Vanderlugt, M. Dal Canto, and S. Miller. 1995. Functional evidence for epitope spreading in the relapsing pathology of experimental autoimmune encephalomyelitis. J. Exp. Med. 182:75-85.

43. Yu, M., J. Johnson, and V. Tuohy. 1996. A predictable sequential determinant spreading cascade invariably accompanies progression of experimental autoimmune encephalomyelitis: a basis for peptide-specific therapy after onset of clinical disease. J. Exp. Med. 183:1777-1788. 
44. Wang, Z.-Y., D. Okita, J. Howard, Jr., and B. Conti-Fine. 1997. TH1 epitope repertoire on the alpha subunit of human muscle acetylcholine receptor in Myasthenia Gravis. Neurology. 48:1643-1653.

45. Chen. Y., V.K. Kuchroo, J. Inobe, D.A. Hafler, and H.L. Weiner. 1994. Regulatory $\mathrm{T}$ cell clones induced by oral tolerance: suppression of autoimmune encephalomyelitis. Science. 265:1237-1240.

46. Gray, D. 1993. Immunological memory. Annu. Rev. Immunol. 11:49-77.

47. Panina-Bordignon, P., A. Tan, A. Termijtelen, S. Demotz, G. Corradin, and A. Lanzavecchia. 1989. Universally immunogenic T cell epitopes: promis- cuous binding to human MHC class II and promiscuous recognition by $\mathrm{T}$ cells. Eur. J. Immunol. 19:2237-2242.

48. Ho, P., D. Mutch, K. Winkel, A.J. Saul, G.L. Jones, T.J. Doran, and C.M. Rzepczyk. 1990. Identification of two promiscuous T cell epitopes from tetanus toxin. Eur. J. Immunol. 20:477-483.

49. Diethelem-Okita, B., R. Raju, D. Okita, and B. Conti-Fine. 1997. Epitope repertoire of human CD41 T cells on tetanus toxin: identification of immunodominant sequence segments. J. Infect. Dis. 175:382-391. 